\title{
Fabrication of Microstructured Optical Fiber (MOF) segments by two- photon lithography 3D printing
}

\author{
Andrea Bertoncini ${ }^{1}$, Carlo Liberale ${ }^{1,2}$ \\ 1. Biological and Environmental Science and Engineering Division, King Abdullah University of Science and Technology, Thuwal, \\ Saudi Arabia \\ 2. Computer, Electrical and Mathematical Science and Engineering Division, King Abdullah University of Science and Technology, \\ Thuwal, Saudi Arabia
}

Microstructured optical fibers (MOFs) have interesting properties such as high birefringence, high nonlinearity, and endlessly single-mode operation [1]. MOFs are optical fibers made with a single material, in which an array of micron-sized longitudinal holes enables the light guidance. The design of the hole pattern allows to control the optical properties of the MOFs.

The prime way to fabricate MOFs is by the drawing of a centimeter-sized preform that mirrors the fiber's final geometry. However, the preform geometry is generally not preserved during the drawing process. During the drawing, several physical phenomena such as material viscosity, gravity, and surface tension, play an essential role in the outcome. In the best scenario, fabricating a designed MOF requires several iteration loops, in which fabrication conditions such as hole pressure, drawing temperature, and preform geometry are optimized. This process typically requires several weeks. In the worst scenarios, fabricating some designed MOFs is just impossible.

Here we show the drawing-less fabrication of MOF segments by high-resolution 3D printing based on twophoton lithography (Nanoscribe GT Photonic professional). This method allows the fabrication of MOF segments with arbitrary geometries. We demonstrate the successful reproduction of different classes of MOFs fabricated with conventional methods, such as solid-core MOFs, anti-resonant hollow-core MOFs, twisted coreless MOFs, photonic band-gap hollow-core MOFs and ring-core MOFs. Our method's unprecedented precision and flexibility allowed us to fabricate the first-ever fiber polarising beam splitter (PBS) based on MOFs. The PBS comprises three parts: a tapered waveguide coupler [2], a dual-core birefringent waveguide, which acts as a directional coupler, and a core spacer to spatially separate the two beams with different polarizations. This device is ultracompact, with a total length of $210 \mu \mathrm{m}$ and integrated on a commercial single-mode fiber.

a) Highly Non Linear
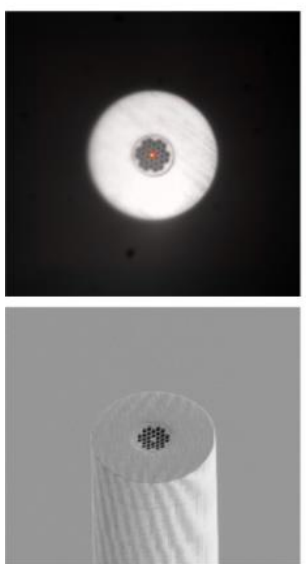

b) Fractal Ring-core
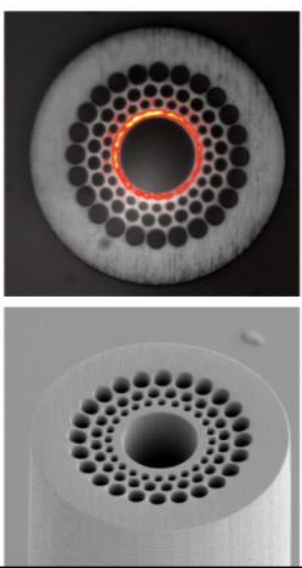

c) Photonic BandGap
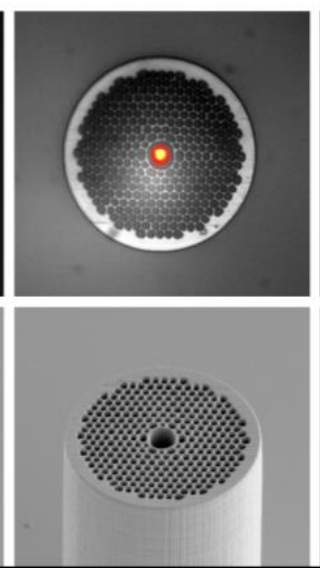

d) Anti-resonant
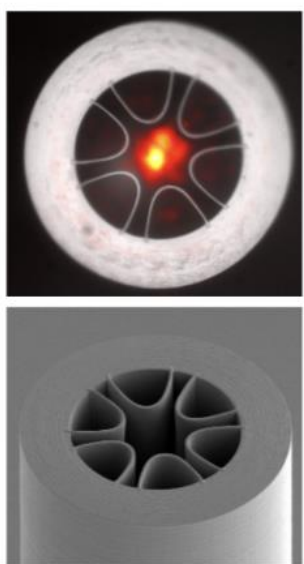

e) Twisted Coreless
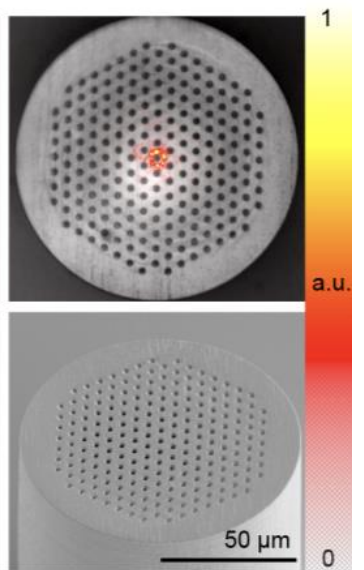

Fig. 1 Set of replicated 3D printed MOFs segments. The top row shows the optical image and measured fiber output beam. The bottom row shows the corresponding images acquired with a Scanning Electron Microscope.

\section{Example References}

[1] P. Russell, "Photonic crystal fibers." Science 299, 358-362 (2003).

[2] A. Bertoncini, V. P. Rajamanickam, and C. Liberale, "On-fiber 3d printing of photonic crystal fiber tapers for mode field diameter conversion," in The European Conference on Lasers and Electro-Optics, (Optical Society of America, 2017), p. CE_6_2. 